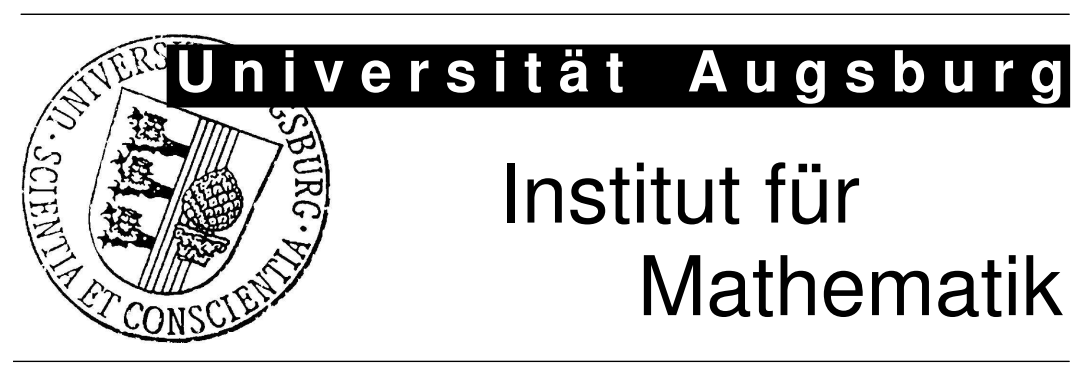

Peter Quast, Makiko Sumi Tanaka

On the Geometry of Symmetric R-Spaces

Preprint Nr. 09/2014 - 02. Dezember 2014

Institut für Mathematik, Universitätsstraße, D-86135 Augsburg

http://www.math.uni-augsburg.de/ 


\section{Impressum:}

\section{Herausgeber:}

Institut für Mathematik

Universität Augsburg

86135 Augsburg

http://www . math. uni-augsburg.de/de/forschung/preprints.html

\section{ViSdP:}

Peter Quast

Institut für Mathematik

Universität Augsburg

86135 Augsburg

Preprint: Sämtliche Rechte verbleiben den Autoren (C) 2014 


\title{
ON THE GEOMETRY OF SYMMETRIC $R$-SPACES
}

\author{
PETER QUAST AND MAKIKO SUMI TANAKA
}

\begin{abstract}
In this survey article we report on our recent work [9, 35], partially in collaboration with Jost-Hinrich Eschenburg, on geometric properties of symmetric $R$-spaces and their submanifolds. But this paper also contains a new result, Theorem 12 , on the convexity of certain reflective submanifolds in simply connected irreducible compact symmetric spaces of Dynkin type $\mathfrak{a}$.
\end{abstract}

\section{Introduction and Preliminaries}

1.1. Riemannian symmetric spaces. Important isometries of a euclidean space $E$ are reflections through affine subspaces. They generate the full isometry group of $E$. A particular reflection is the symmetry $s_{p}$ through a point $p \in E$. It reverses the orientation of oriented straight lines (geodesics) emanating in $p$. Analogously, if $S$ is a connected Riemannian manifold and $p$ a point in $S$, an isometry $s_{p}$ of $S$ that fixes $p$ and reverses the orientations of all geodesics emanating in $p$ is called (geodesic) symmetry of $S$ through $p$. A generic Riemannian manifold does, of course, not admit any geodesic symmetry.

A (Riemannian) symmetric space is a connected Riemannian manifold $S$ such that for each point $p \in S$ the geodesic symmetry $s_{p}$ exists. Locally symmetric spaces are characterized by the property that their Riemannian curvature tensor is covariantly constant (parallel). From this point of view symmetric spaces are generalizations of euclidean space. Symmetric spaces were introduced by Élie Cartan in the 1920s (see [3, Chap. IV] and [1, $\S 6.7-\S 6.9]$ for interesting historical accounts). Classical references on symmetric spaces include Sigurdur Helgason's monograph [16] and Ottmar Loos' two volumes [24, 25]. We refer to these books for further details and proofs.

To a symmetric space $S$ one associates two transitively acting closed subgroups of the full isometry group $\operatorname{Iso}(S)$ : the symmetry group $\operatorname{Sym}(S)$ generated by all geodesic symmetries, and the transvection group $\operatorname{Trans}(S)$ generated by compositions of two geodesic symmetries. If $S$ is compact, the transvection group of $S$ is actually the identity component of $\operatorname{Iso}(S)$. We choose a base point $o \in S$ and denote by $K$ the identity component of the isotropy group $\{g \in \operatorname{Iso}(S) \mid g(o)=o\}$. The group $K$ acts effectively on $T_{o} S$. This action is called isotropy representation. A symmetric space is called irreducible, if its isotropy representation is irreducible. Further we say that a symmetric space is of compact type, if its universal Riemannian cover is still compact.

2010 Mathematics Subject Classification. Primary 53C35; Secondary 53C40.

Key words and phrases. symmetric $R$-spaces, extrinsic symmetry, submanifolds, unit lattice, convexity.

This article is for the proceedings of the Workshop "Development of group actions and submanifold theory" (June 2014) at RIMS, Kyoto University. It will appear in RIMS Kôkyûroku. 
An important tool for studying symmetric spaces are flats. A flat $F$ of a symmetric space $S$ is a maximal connected complete totally geodesic submanifold $F \subset S$ of vanishing sectional curvature. Any two flats of a symmetric space $S$ can be identified by an isometry of $S$. Thus the dimension of any two flats of $S$ coincide. This dimension is called the rank of $S, \operatorname{rank}(S)=\operatorname{dim}(F)$.

If a symmetric space $S$ is compact, then any flat of $S$ is a flat torus. The unit lattice of a compact symmetric space $S$ is the unit lattice of one of its flats. Let $F$ be a flat of $S$ and let $o \in F$, then the unit lattice of $S$ with respect to $F$ and $o$ is

$$
\Gamma=\Gamma\left(T_{o} F\right):=\left\{X \in T_{o} F \mid \operatorname{Exp}_{o}(X)=o\right\},
$$

where $\operatorname{Exp}_{o}: T_{o} S \rightarrow S$ denotes the Riemannian exponential map of $S$ with respect to the point $o$. We say that $\Gamma$ is rectangular (resp. cubic), if there exists an orthogonal (resp. orthonormal) basis $\left\{e_{1}, \ldots, e_{r}\right\}$ of $T_{o} F$ such that

$$
\Gamma=\operatorname{spann}_{\mathbb{Z}}\left(e_{1}, \ldots, e_{r}\right)=\left\{\sum_{j=1}^{r} \lambda_{j} e_{j} \mid \lambda_{1}, \ldots, \lambda_{r} \in \mathbb{Z}\right\} .
$$

1.2. Symmetric $R$-spaces. Symmetric $R$-spaces were introduced by Tadashi Nagano [31] and Masaru Takeuchi [39] in 1965 as compact symmetric spaces which are at the same time $R$-spaces. This means that they also admit a transitive action of a centre-free non-compact semisimple Lie group and the corresponding stabilizer of a point is a certain maximal parabolic subgroup. For a geometric interpretation of this non-compact transformation group of a symmetric $R$-space we refer to [42] and [15]. We call a symmetric $R$-space indecomposable, if it is not a (global) Riemannian product of two symmetric $R$-spaces. Symmetric $R$-spaces appear in various geometric contexts.

Shoshichi Kobayashi and Tadashi Nagano classified symmetric $R$-spaces in [18. It turns out that every indecomposable symmetric $R$-space $P$ can be obtained as follows: Let $S$ be a simply connected irreducible compact symmetric space and let $o \in S$. We take an element $\xi \in T_{o} S$ such that the linear operator $T_{o} S \rightarrow T_{o} S, X \mapsto R(\xi, X) \xi$ has precisely spectrum $\{0,-1\}$. Those elements $\xi$ are called extrinsically symmetric. The extrinsically symmetric elements in $T_{o} S$ can be read off from the Satake diagram (see [18, Section 6]) or from the Dynkin diagram (see e.g. [28, Lemma 2.1]) of $S$. Every connected component of the set of all extrinsically symmetric elements in $T_{o} S$ is an orbit of the isotropy representation and an indecomposable symmetric $R$-space. Vice-versa, every indecomposable symmetric $R$-space is obtained in this way (see [18, 19] and also [17, 40]).

Using an algebraic description of symmetric $R$-spaces in terms of so called compact Jordan triple systems, Ottmar Loos characterized symmetric $R$-spaces among all compact symmetric spaces as those whose metric on irreducible factors can be rescaled in such a way that the unit lattice gets cubic (see [26, 27]).

An important subclass of symmetric $R$-spaces are the hermitian symmetric spaces of compact type. A hermitian symmetric space is a symmetric space that also carries a Kähler structure such that all geodesic symmetries are holomorphic. Moreover, Masaru Takeuchi showed that every real form (that is a totally real totally geodesic submanifold 
of half dimension) of a hermitian symmetric space of compact type is a symmetric $R$-space and that every symmetric $R$-space can be realized in such a way (see [41] and also [23], [45, proof of Theorem 4.3] and [36]).

Raoul Bott used symmetric $R$-spaces to prove his famous periodicity theorem for the stable homotopy of unitary, orthogonal and symplectic groups (see [4] and [30, Sections 23, $24]$ ). There is also a periodicity result for some typical embeddings of symmetric $R$-spaces into each other (see [29]).

1.3. Extrinsically symmetric spaces. A connected submanifold $P \subset E$ of a euclidean space $E$ is called an extrinsically symmetric space if for all $p \in P$ the submanifold $P$ is invariant under the reflections $\rho_{p} \in \operatorname{Iso}(E)$ through the affine normal space of $p+N_{p} P$ of $P$ at $p$.

While Riemannian symmetric spaces are locally characterized by the parallelism of their Riemannian curvature tensors, extrinsic symmetric spaces are characterized by the parallelism of their second fundamental form $\alpha$ (w.r.t. the induced connection on the normal bundle). Unlike in the case of Riemannian symmetric spaces, the parallelism of the second fundamental form $\alpha$ of $P \subset E$ characterizes extrinsically symmetric spaces globally, if one assumes that $P$ is connected and complete, as shown by Wolf Strübing (see [38] and [14]). Moreover, for compact submanifolds of euclidean space this characterization is stable in the sense that compact submanifolds of euclidean space with almost parallel second fundamental form are just small deformations of extrinsically symmetric ones (see [34]).

Dirk Ferus classified extrinsically symmetric spaces by showing that every extrinsically symmetric space is a product of a compact extrinsically symmetric space and an affine subspace. Further, every compact extrinsically symmetric space is a symmetric $R$-space, realized as a connected component of the set of extrinsically symmetric elements in the tangent space of some symmetric space of compact type (see [12, 14] and also [7]). Viceversa, every symmetric $R$-space realized in this manner is extrinsically symmetric (see [11, 14]).

By the very definition of an extrinsically symmetric space $P \subset E$, any element $f \in$ $\operatorname{Sym}(P)$ is the restriction to $P$ of an isometry $\hat{f}$ of $E$. Recently Jost-Hinrich Eschenburg and the authors have shown that this holds for any isometry of $P$ :

Theorem 1 ([8, 10]). Every isometry $f$ of a compact extrinsically symmetric space $P \subset E$ is the restriction of a linear ${ }^{1}$ isometry $\hat{f}$ of $E$.

Unfortunately, in the case of non-hermitian extrinsically symmetric spaces our proof uses the classification of compact extrinsically symmetric spaces and a case-by-case verification.

A beautiful standard reference for extrinsically symmetric spaces and related topics is [2].

1.4. Intrinsically and extrinsically reflective submanifolds. A reflective submanifold $M$ in a Riemannian manifold $N$ is a connected component of the fixed point set of

\footnotetext{
${ }^{1}$ We may always assume that the barycentre of $P$ is the origin of $E$.
} 
an involutive isometry $\sigma$ of $N$. This isometry $\sigma$ will be called reflection of $N$ through $M$. Reflective submanifolds are automatically totally geodesic. Reflective submanifolds in symmetric spaces have been studied and classified by Dominic S. P. Leung in the series of papers [20, 21, 22, 23]. They include important totally geodesic submanifolds such as polars, meridians (see below) and centrioles, which were introduced and extensively studied by Bang-Yen Chen and Tadashi Nagano and their students (see e.g. [5, 6] or [32]).

A totally geodesic submanifold $M \subset N$ of a submanifold $N \subset E$ of a euclidean space $E$ is called extrinsically reflective, if $M$ is a connected component of the intersection of $N$ with the fixed set of an involutive isometry of $E$ that leaves $N$ invariant.

Proposition 2 ([9, Theorem 2]). An extrinsically reflective submanifold $M \subset P$ of an extrinsically symmetric space $P \subset E$ is extrinsically symmetric in $E$.

Observation 3. In view of Theorem 1, every reflective submanifold of a compact extrinsically symmetric space is actually extrinsically reflective, and thus extrinsically symmetric. In other words, any reflective submanifold of a symmetric $R$-space is a symmetric $R$-space. This generalizes a claim in [43, Lemma 3.1].

1.5. Meridians. Let $S$ be a compact symmetric space. We choose an origin $o \in S$ and a fixed point $p \in S$ of the geodesic symmetry $s_{o}$ with $p \neq o$ Then the geodesic symmetries $s_{o}$ and $s_{p}$ commute. The meridian of $S$ corresponding to $o$ and $p$, often denoted by $S_{-}$, is the connected component of the fixed point set of the involutive isometry $s_{o} \circ s_{p}$ that contains $p$. The terminology 'meridian' was introduced by Bang-Yen Chen and Tadashi Nagano in [5].

Remark. If $p$ is an isolated fixed point of $s_{o}$, then $s_{p}=s_{o}$ and $s_{o} \circ s_{p}=$ id and the corresponding meridian $S_{-}$coincides with $S$. Unless $p$ is an isolated fixed point of $s_{o}$ we have $\operatorname{dim}\left(S_{-}\right)<\operatorname{dim}(S)$.

Proposition 4 ([5, Lemma 2.3]). The rank of a compact symmetric space $S$ coincides with the rank of any of its meridians $S_{-}$, that is $\operatorname{rank}\left(S_{-}\right)=\operatorname{rank}(S)$. In particular, any flat of $S_{-}$is also a flat of $S$.

If $S$ is a compact extrinsically symmetric space in $E$, then the involutive isometry $s_{o} \circ s_{p}$ is the restriction to $S$ of the linear isometry $\rho_{o} \circ \rho_{p}$. If we assume that $S$ is full in $E$, then $\rho_{o} \circ \rho_{p}$ is has order two. With Proposition 2 we conclude:

Observation 5. Any meridian $P_{-}$of a compact extrinsically symmetric space $P \subset E$ is itself extrinsically symmetric in $E:^{2}$

\section{The Unit LATTICE OF COMPACT EXTRINSICALly SYMMETRIC SPACES}

In this section we report on our recent work [9] joint with Jost-Hinrich Eschenburg.

Using algebraic techniques Ottmar Loos [26, 27] proved that symmetric $R$-spaces are precisely the compact symmetric spaces whose unit lattice is cubic, after a suitable rescaling

\footnotetext{
${ }^{2}$ Although this is just a special case of Observation 3, our proof does not use classification and case-bycase verification.
} 
of the metric on irreducible factors. In [9] Jost-Hinrich Eschenburg and the authors gave a purely differential geometric proof of the following statement originally due to Ottmar Loos:

Theorem 6 ([26, 27]). The unit lattice of a compact extrinsically symmetric space $P \subset E$ is rectangular.

Although this fact is well known, we think that some methods used in [9] might still be interesting. A first statement for which we provide a detailed and elementary proof concerns extrinsically symmetric flat tori:

Theorem 7 ([14, Theorem 3], [9, Theorem 3]). A full d-dimensional extrinsically symmetric flat torus $F \subset E$ is an extrinsic product torus. This means that $F$ is a Riemannian product of planar round circles $S^{1}\left(r_{i}\right) \subset E_{i}, i=1, \ldots, d$, of possibly different radii $r_{i}$ in affine 2-dimensional subspaces of $E_{i} \subset E$, which are perpendicular to each other ${ }^{3}$. In particular, the unit lattice of $F$ is rectangular.

Proof. This proof is a detailed elaboration of the arguments given [14] and [9].

Let $f: \mathbb{R}^{d} \rightarrow E$ be the isometric immersion given by the universal covering of $F$. By $\partial_{i}$ we denote the partial derivative operator $\frac{\partial}{\partial x_{i}}$. Similarly $\partial_{i j}$ and $\partial_{i j k}$ denote the operators $\frac{\partial^{2} f}{\partial x_{i} \partial x_{j}}$ and $\frac{\partial^{3} f}{\partial x_{i} \partial x_{j} \partial x_{k}}$ respectively. Notice that $\left\{\partial_{i} f \mid i=1, \ldots, d\right\}$ is an orthonormal tangent frame on $F$. Since constant vector fields on $\mathbb{R}^{d}$ are parallel and $f$ is an isometric immersion, the tangent vectors fields $\partial_{i} f$ on $F$ are parallel, too. Thus $\partial_{i j} f=\alpha\left(\partial_{i} f, \partial_{j} f\right)=: \alpha_{i j}$ is a normal vector field on $F$ for any $i, j \in\{1, \ldots, d\}$.

As $F$ is extrinsically symmetric, its second fundamental form $\alpha$ is parallel. By fullness, the normal space of $F$ is generated by the $\alpha_{i j}, i, j \in\{1, \ldots, d\}$. Moreover $\partial_{i j k} f=-A_{\alpha_{i j}} \partial_{k} f$ are tangent vector fields on $F$.

We observe that the linear endomorphisms $A_{\alpha_{i j}}$ are parallel and commute with each other. Thus there is an orthogonal decomposition $T F=E_{1} \oplus \cdots \oplus E_{r}, r \leq d$, of $T F$ into parallel common eigendistributions of the $A_{\alpha_{i j}}$. We may assume that the parallel tangent vector fields $\partial_{i} f$ are common eigenvectors of the $A_{\alpha_{i j}}$. Thus we get $\partial_{i j k} f=-A_{\alpha_{i j}} \partial_{k} f=$ $\lambda_{i j k} \partial_{k} f$. The eigenvalues $\lambda_{i j k}$ are constant, because the endomorphisms $A_{\alpha_{i j}}$ are parallel. Since the partial derivatives commute, we see that the eigenvalues $\lambda_{i j k}$ must vanish, if at least two indices $i, j, k$ are distinct. The only possible non-zero eigenvalues are therefore $\lambda_{i}:=\lambda_{i i i}$. We are left with the differential equations $\partial_{i i i} f=\lambda_{i} \partial_{i} f$ for $i=1, \ldots, d$. From $\left\langle\alpha_{i j}, \alpha_{i j}\right\rangle=\left\langle A_{\alpha_{i j}} \partial_{i} f, \partial_{j} f\right\rangle=0$ if $i \neq j$, we conclude that $\alpha_{i j}=\partial_{i j} f=0$ for $i \neq j$. In a similar way we observe that $\alpha_{i i}$ is everywhere perpendicular to $\alpha_{j j}$ for $i \neq j$.

Summing up we get the following system of differential equations

$$
\begin{array}{rll}
\partial_{i i i} f-\lambda_{i} \partial_{i} f & =0 & \text { for } i=1, \ldots, d, \\
\partial_{i j} f=0 & \text { for } i \neq j .
\end{array}
$$

\footnotetext{
${ }^{3}$ Although the radii of the planar round circles can be distinct, such a torus is sometimes still called a Clifford torus.
} 
Solving Equation (1) yields

$$
\partial_{i} f= \begin{cases}c_{i 1}+c_{i 2} \cdot x_{i}, & \text { if } \lambda_{i}=0 \\ c_{i 1} \exp \left(\sqrt{\lambda_{i}} x_{i}\right)+c_{i 2} \exp \left(-\sqrt{\lambda_{i}} x_{i}\right), & \text { if } \lambda_{i}>0 \\ c_{i 1} \sin \left(\sqrt{-\lambda_{i}} x_{i}\right)+c_{i 2} \cos \left(\sqrt{-\lambda_{i}} x_{i}\right), & \text { if } \lambda_{i}<0\end{cases}
$$

where the functions $c_{i 1}: \mathbb{R}^{d} \rightarrow E$ and $c_{i 2}: \mathbb{R}^{d} \rightarrow E$ do not depend on the $i$-th variable $x_{i}$. But, by Equation (2), the functions $\partial_{i} f: \mathbb{R}^{d} \rightarrow E$ only depend on $x_{i}$. Thus $c_{i 1}$ and $c_{i 2}$ are constant functions.

Since $\partial_{i} f$ has everywhere length one, only the following two cases are possible:

$$
\partial_{i} f= \begin{cases}c_{i 1}, & \text { if } \lambda_{i}=0 \\ c_{i 1} \sin \left(\sqrt{-\lambda_{i}} x_{i}\right)+c_{i 2} \cos \left(\sqrt{-\lambda_{i}} x_{i}\right), & \text { if } \lambda_{i}<0\end{cases}
$$

By integrating $\partial_{i} f$ in the direction of $x_{i}$ we see that only $\lambda_{i}<0$ for all $i \in\{1, \ldots, d\}$ can occur, because $f\left(\mathbb{R}^{d}\right)=F$ is compact; that is

$$
\partial_{i} f=c_{i 1} \sin \left(\sqrt{-\lambda_{i}} x_{i}\right)+c_{i 2} \cos \left(\sqrt{-\lambda_{i}} x_{i}\right) .
$$

In particular no $\alpha_{i i}$ has zeros. Thus the set $\left\{\partial_{i} f \mid i=1, \ldots, d\right\} \cup\left\{\partial_{i i} f \mid i=1, \ldots, d\right\}$ is at each point an orthogonal basis of $E$. In particular $\operatorname{dim}(E)=2 d$. As $\partial_{i} f$ has constant length one, $c_{i 1}$ and $c_{i 2}$ are unit vectors.

Recall that $\partial_{i} f$ is everywhere perpendicular to $\partial_{j} f$ for $i \neq j$. By taking appropriate values for $x_{i}$ and $x_{j}$, we observe that $c_{i k}$ is perpendicular to $c_{j l}$ for $i \neq j$ and $k, l \in\{1,2\}$. Moreover, since $\partial_{i} f$ and

$$
\partial_{i i} f=c_{i 1} \sqrt{-\lambda_{i}} \cos \left(\sqrt{-\lambda_{i}} x_{i}\right)-c_{i 2} \sqrt{-\lambda_{i}} \sin \left(\sqrt{-\lambda_{i}} x_{i}\right)
$$

are everywhere perpendicular, we see, if we take $x_{i}=0$ and $x_{i}=\frac{\pi}{2 \sqrt{-\lambda_{i}}}$, that the unit vectors $c_{i 1}$ and $c_{i 2}$ are perpendicular. Summing up, $\left\{c_{i 1} \mid i=1, \ldots, d\right\} \cup\left\{c_{i 2} \mid i=1, \ldots, d\right\}$ is an orthonormal basis of $E$.

By integration we get

$$
f\left(x_{1}, \ldots, x_{d}\right)=v+\sum_{i=1}^{d} \frac{1}{\sqrt{-\lambda_{i}}}\left(-\cos \left(\sqrt{-\lambda_{i}} x_{i}\right) c_{i 1}+\sin \left(\sqrt{-\lambda_{i}} x_{i}\right) c_{i 2}\right)
$$

for some $v \in E$. This shows that $F=f\left(\mathbb{R}^{d}\right)$ is an extrinsic product torus.

Sketch of proof of Theorem 6. We are now able to sketch our proof of Theorem 6 . Using Proposition 4, we lower the dimension of the submanifold while keeping the rank by the following iteration: Starting with $P \subset E$, we take a meridian $P_{-}$of $P$. By Observation 5 $P_{-}$is again extrinsically symmetric in $E$. Next we consider a meridian of $P_{-}$and then take meridians again and again, until we reach a fixed point of this iteration scheme. This fixed point must be a compact extrinsically symmetric space all of whose geodesic symmetries only have isolated fixed points. In other words, we could have assumed right away that the geodesic symmetries of our compact extrinsically symmetric space $P \subset E$ only have isolated fixed points.

Considering a compact covering where all euclidean factors split off, we could show: 
Proposition 8 ([9, Lemma 7]). A compact symmetric space $P$ all of whose geodesic symmetries only have isolated fixed points is a Riemannian product of a simply connected symmetric space $P^{\prime}$ of compact type and possibly a flat torus $T$, that is $P=P^{\prime} \times T$.

If the root system of a simply connected irreducible symmetric space $P^{\prime}$ of compact type has not type $\mathfrak{a}_{1}$, then there is a closed geodesic $\gamma$ in $P^{\prime}$ admitting a Jacobi-field $J$ that vanishes at the starting point $o=\gamma(0)$ of $\gamma$ but not at the antipodal point $p$ of $o$ in the circle $\gamma$. Looking at the variation of closed geodesics defined by $J$, we see that the connected component of $\operatorname{Fix}\left(s_{o}\right) \subset P^{\prime}$ that contains $p$ has positive dimension. This yields:

Theorem 9 ([9, Theorem 9]). The only simply connected irreducible symmetric spaces of compact type whose geodesic symmetries only have isolated fixed points are round spheres.4

At this point we may assume that our compact extrinsically symmetric space $P \subset E$ is intrinsically a Riemannian product of $k$ round spheres $\mathbb{S}_{1}, \ldots, \mathbb{S}_{k}$ and possibly a flat torus $T$, that is

$$
P=\mathbb{S}_{1} \times \cdots \times \mathbb{S}_{k} \times T .
$$

Obviously, a maximal torus of $P$ has the form

$$
F=C_{1} \times \cdots \times C_{k} \times T,
$$

where $C_{j}$ is a great circle in $\mathbb{S}_{j}$ for $j=1, \ldots, k$, and it is a reflective submanifold of $P 5^{5}$ Theorem 6 follows directly from Theorem 7 if $k=0$. If $P$ contains even-dimensional spheres, we split them off as follows: Assume w.l.g. that $\mathbb{S}_{1}$ has even dimension and set $P^{\prime}:=\mathbb{S}_{2} \times \cdots \times \mathbb{S}_{k} \times T$. We choose a point $x_{1} \in \mathbb{S}_{1}$. The geodesic symmetry $s_{x_{1}}$ of $\mathbb{S}_{1}$ at $x_{1}$ lies in the transvection group of $\mathbb{S}_{1}$. Therefore the involution $s_{x_{1}} \times \operatorname{id}_{P^{\prime}}$ lies in the identity component of $\operatorname{Iso}(P)$, which is Trans $(P)$. Thus $s_{x_{1}} \times \mathrm{id}_{P^{\prime}}$ extends to an extrinsic reflection, and the connected component $\left\{x_{1}\right\} \times P^{\prime} \cong P^{\prime}$ of $\operatorname{Fix}\left(s_{x_{1}} \times \operatorname{id}_{P^{\prime}}\right)$ is extrinsically symmetric by Proposition 2. We are left to show that $P^{\prime}$ has a rectangular unit lattice. Applying the above argument recursively, we may assume that all sphere factors of $P^{\prime}$ have odd dimensions.

In other words, we may assume that our extrinsically symmetric space $P \subset E$ is intrinsically a Riemannian product

$$
P=\mathbb{S}_{1} \times \cdots \times \mathbb{S}_{k} \times T
$$

of odd dimensional round spheres $\mathbb{S}_{1}, \ldots, \mathbb{S}_{k}$ and perhaps a flat torus $T$. For each $j \in$ $\{1, \ldots, k\}$ we now choose a great circle $C_{j}$ in $\mathbb{S}_{j}$. The reflection $r_{j}$ of $\mathbb{S}_{j}$ through $C_{j}$ is a transvection of $\mathbb{S}_{j}$, since $\mathbb{S}_{j}$ has odd dimension. Thus the involutive isometry $r_{1} \times \cdots \times$ $r_{k} \times \mathrm{id}_{T}$ is a transvection of $P$ and therefore extends to an extrinsic involutive isometry. With Proposition 2 we conclude that the maximal torus $F=C_{1} \times \cdots \times C_{k} \times T$ of $P$ is extrinsically symmetric and has rectangular unit lattice by Theorem 7 .

\footnotetext{
${ }^{4}$ This was already known before as a consequence of the classification of polars in compact symmetric spaces (these are connected components of the fixed point set of a geodesic symmetry) due to Bang-Yen Chen and Tadashi Nagano (see [5, 6, 32]). But our proof is purely conceptional.

${ }^{5}$ If one uses Observation 3 (for which we unfortunately only know a proof using classification and which is therefore not in the spirit of [9]), then Theorem 6 follows directly from Theorem 7 .
} 
Question. At RIMS Workshop we were asked by Professor Yoshihiro Ohnita if our method can be adapted to show that the unit lattice of an indecomposable symmetric $R$-space is actually cubic. Unfortunately, we cannot answer this question $\sqrt{6}$

\section{Convexity of Reflective submanifolds of Symmetric $R$-SPACES}

In this section we give an overview of our work published in [35].

A geodesically complete submanifold $M \subset N$ in a Riemannian manifold $N$ is called (geodesically) convex, if the Riemannian distance between any two points $m_{1}, m_{2} \in M$ measured within $M$ coincides with the Riemannian distance between $m_{1}$ and $m_{2}$ measured within $N$, or, equivalently, if any shortest geodesic arc in $M$ is still shortest in $N$. One might think of convexity as a 'global version' of being totally geodesic.

Reflective submanifolds certainly form a very important class of totally geodesic submanifolds of compact symmetric spaces. One may therefore wonder whether reflective submanifolds in compact symmetric spaces are geodesically convex. Already in the fairly easy example of a flat 2-torus $\mathbb{R}^{2} / \Gamma$, whose unit lattice $\Gamma$ is rhombic and not rectangular, the long diagonal is an example of a reflective but non-convex submanifold. This is bad news! Indeed, adapting results of Takashi Sakai [37] on the cut locus of compact symmetric spaces, Hiroyuki Tasaki showed that convexity is already detected on the level of flats.

Proposition 10 ([46, Lemma 2.2]). Let $M \subset S$ be a reflective submanifold in a compact symmetric space $S$. Let $F_{M}$ be a flat of $M$ and $F_{S}$ a flat of $S$ containing $F_{M}$, that is $F_{M} \subset F_{S}$. Then $M$ is convex in $S$ if and only if $F_{M}$ is convex in $F_{S}$.

It turns out that the situation described above appears in the symmetric space $S=$ $\mathrm{SU}_{3} / \mathbb{Z}_{3}$, whose unit lattice is rhombic. The flat $F_{M}$ of the reflective submanifold $M$ which isomorphic to $\mathrm{SO}_{3}$ in $S=\mathrm{SU}_{3} / \mathbb{Z}_{3}$ is the long diagonal in $F_{S}$ (see [35]). This example works in all dimensions: The reflective submanifold given by the complex conjugation in $S=\mathrm{SU}_{n} / \mathbb{Z}_{n}$ is never convex for $n \geq 3$. On the other hand, Felix Platzer and the first author have shown using case-by-case arguments:

Theorem 11 ([33]). Every reflective submanifold in a special unitary group $\mathrm{SU}_{n}$ is convex.

As a consequence we get:

Theorem 12. Let $S$ be a simply connected irreducible symmetric space of compact type and of rank $r \geq 2$, whose root system is of type $\mathfrak{a}_{r} \cdot{ }^{7}$ Let $M \subset S$ be a reflective submanifold that has the following property:

(*) There exists $\xi \in T_{o} F_{M} \subset T_{o} F_{S}$ which is regular w.r.t. the root system of $S:^{8}$

\footnotetext{
${ }^{6}$ At this point we recall that Dirk Ferus has shown in [13] that the shape operator in the direction of the mean curvature vector field of a compact indecomposable extrinsically symmetric space is a multiple of the identity.

7 This means that $S$ is $\mathrm{SU}_{r+1}$ or $\mathrm{SU}_{r+1} / \mathrm{SO}_{r+1}$ or $\mathrm{SU}_{2 r+2} / \mathrm{Sp}_{r+1}$ or $\mathrm{E}_{6} / \mathrm{F}_{4}$ (see e.g. [16, Chapter X]).

${ }^{8}$ Here we use the notation introduced in Proposition 10 with $o \in F_{M}$. A vector $\xi \in T_{o} F_{S}$ is called regular w.r.t. the root system of $S$, if $\alpha(\xi) \neq 0$ for all roots $\alpha$ of $S$. For details on root systems of symmetric spaces we refer to the standard literature such as [25] or [16]. An equivalent formulation of condition $(*)$ is that $F_{S}$ is the unique extension of $F_{M}$ to a flat of $S$.
} 
Then $M$ is convex in $S$.

Proof. Set $\mathfrak{t}:=T_{o} F_{S}$ and $\mathfrak{a}:=T_{o} F_{M} \subset \mathfrak{t}$ for short. Choose a maximal abelian subspace $\hat{\mathfrak{t}}$ of the Lie algebra $\mathfrak{s u}_{r+1} \cong T_{I} \mathrm{SU}_{r+1}$ and denote by $F_{\hat{S}}$ the flat of the special unitary group $\hat{S}:=\mathrm{SU}_{r+1}$ satisfying $\hat{\mathfrak{t}}=T_{I} F_{\hat{S}}$. Since the symmetric spaces $S$ and $\hat{S}$ both have rank $r$ and root systems of type $\mathfrak{a}_{r}$, there exists (for a suitably scaled bi-invariant metric on $\hat{S}$ ) an orthogonal linear map $\iota_{*}: \mathfrak{t} \rightarrow \hat{\mathfrak{t}}$ that identifies the root system of $S$ with the root system of $\hat{S}$. Since the unit lattice of a simply connected irreducible symmetric space is generated by its system of inverse roots (see e.g. [25, pp. 25, 69, 77]), which in the case $\mathfrak{a}_{r}$ coincides with the set of root vectors, $\iota_{*}$ induces an isometry $\iota: F_{S} \rightarrow F_{\hat{S}}$ with $\iota(o)=e$.

The reflection $\sigma$ of $S$ through $M$ leaves $F_{S}$ invariant (see [44, Lemma 3.1], 35, Observation 4]), and its differential $\sigma_{*}: \mathfrak{t} \rightarrow \mathfrak{t}$ restricted to $\mathfrak{t}=T_{o} F_{S}$ is an involutive orthogonal map that leaves the root system of $S$ invariant. Consider

$$
\hat{\sigma}_{*}:=\iota_{*} \circ \sigma_{*} \circ \iota_{*}^{-1}: \hat{\mathfrak{t}} \rightarrow \hat{\mathfrak{t}} .
$$

This is an involutive orthogonal map that leaves the root system of $\hat{S}$ invariant and satisfies $\hat{\mathfrak{a}}:=\operatorname{Fix}\left(\hat{\sigma}_{*}\right)=\iota_{*}(\mathfrak{a})$. Moreover, by condition $(*), \hat{\sigma}_{*}$ fixes the regular element $\hat{\xi}:=\iota_{*}(\xi) \in \hat{\mathfrak{a}}$ and therefore the Weyl chamber $\hat{\mathfrak{t}}^{+}$in $\hat{\mathfrak{t}}$ that contains $\hat{\xi}$. Thus $\hat{\sigma}_{*}$ leaves the system of positive simple roots of $\mathfrak{s u}_{r+1}$ defining $\hat{\mathfrak{t}}^{+}$invariant. Since $\hat{S}=\mathrm{SU}_{r+1}$ is a simply connected simple compact Lie group, $\hat{\sigma}_{*}$ induces an involutive Lie group automorphism $\hat{\sigma}$ of $\hat{S}=\mathrm{SU}_{r+1}$ that leaves $F_{\hat{S}}$ invariant (see [25, Proposition 3.4, p. 128]).

Let $\hat{M} \subset \hat{S}$ be the connected component of $\operatorname{Fix}(\hat{\sigma})$ containing the identity $I$. We claim that $F_{\hat{M}}:=\iota\left(F_{M}\right) \subset F_{\hat{S}}$ is a flat of $\hat{M}$. Indeed, let $\hat{\mathfrak{a}}^{\prime}$ be a maximal abelian subspace of $T_{I} \hat{M} \subset \mathfrak{s} \mathfrak{u}_{r+1}$ that contains $\hat{\mathfrak{a}}$. By condition $(*), \hat{\xi}:=\iota_{*}(\xi) \in \hat{\mathfrak{a}}$ is a regular element w.r.t. the root system of $\hat{S}$. Thus $\hat{\mathfrak{t}}$ is the unique maximal abelian subset of $\mathfrak{s u}_{r+1}$ that contains $\hat{\xi}$ and therefore also the unique maximal abelian subset of $\mathfrak{s u}_{r+1}$ that contains $\hat{\mathfrak{a}}^{\prime}$. But the intersection of $\mathfrak{t}$ with $T_{I} \hat{M}$ is $\hat{\mathfrak{a}}$. Hence $\hat{\mathfrak{a}}^{\prime}=\hat{\mathfrak{a}}$ and $\iota\left(F_{M}\right)$ is a flat of $\hat{M}$.

The reflective submanifold $\hat{M}$ is convex in $\mathrm{SU}_{r+1}$ by Theorem 11, and thus $F_{\hat{M}}$ is convex in $F_{\hat{S}}$. Since $\iota$ is an isometry, $F_{M}$ is also convex in $F_{S}$. Proposition 10 eventually implies the claim.

Now the following question arises:

Question. Can one drop the somewhat technical condition (*) in Theorem 12.

More generally on may ask:

Question. How can one describe the class formed by the compact symmetric spaces all of whose reflective submanifolds are convex?

The authors have shown that the symmetric $R$-spaces form a subclass of this class:

Theorem 13 ([35]). Every reflective submanifold in a symmetric $R$-space is convex.

Outline of proof. Let $\sigma$ be an involutive isometry of a symmetric $R$-space $P$ and let $M$ be a connected component of its fixed point set. In view of Proposition 10 we take a flat $F_{M}$ 
of $M$ and a flat $F_{S}$ of $S$ with $F_{M} \subset F_{S}$. Recall that $\sigma$ leaves $F_{S}$ invariant (see [44, Lemma 3.1], [35, Observation 4]). We have to show that $F_{M}$ is convex in $F_{S}$. For this we choose an origin $o \in F_{M}$. Then we have to prove that

$$
d_{F_{M}}(o, x)=d_{F_{S}}(o, x)
$$

holds for all $x \in F_{M}$, where $d_{F_{M}}$ and $d_{F_{S}}$ denote the Riemannian distances in $F_{M}$ and $F_{S}$ respectively.

As a consequence of Theorem 6 one can show that there exists an orthogonal basis $B=\left\{e_{1}, \ldots, e_{r}\right\}$ of $T_{o} F_{S}$ such that

- the unit lattice $\Gamma$ of $S$ is generated by $B$, that is

$$
\Gamma=\left\{\sum_{j=1}^{r} \lambda_{j} e_{j} \mid \lambda_{j} \in \mathbb{Z}, j=1, \ldots, r\right\} ;
$$

- there exists $p \in\{1, \ldots, r\}$ and $q \in\{2 p, \ldots, r\}$ such that

$$
T_{o} F_{M}=\left\{\sum_{j=1}^{r} \lambda_{j} e_{j} \mid \lambda_{2 j-1}=\lambda_{2 j} \text { for } 1 \leq j \leq p \text { and } \lambda_{q+1}=\cdots=\lambda_{r}=0\right\}
$$

(see [44, Proposition 3.3] for a differential geometric proof and [35, Proposition 5] for an elementary linear algebraic one).

Given $x \in F_{M}$ we take an element $X \in T_{o} F_{M}$ such that $x=\operatorname{Exp}_{o}(X)$, where $\operatorname{Exp}_{o}$ denotes the Riemannian exponential map of $P$ at $o$. Then $d_{F_{S}}(o, x)=\min _{Y \in \Gamma}\|X+Y\|$. One easily constructs an element $Z \in T_{o} F_{M} \cap \Gamma$ such that $\|X+Z\|=\min _{Y \in \Gamma}\|X+Y\|$. We conclude that $d_{F_{M}}(o, x)=d_{F_{S}}(o, x)$.

\section{ACKNOWLEDGEMENTS}

The authors wish to thank Jost-Hinrich Eschenburg and Ernst Heintze for helpful discussions.

\section{REFERENCES}

[1] M. A. Akivis \& B. A. Rosenfeld, Élie Cartan (1869-1951), Translations of Mathematical Monographs, Vol. 123, American Mathematical Society, Providence 1993

[2] J. Berndt, S. Console \& C. Olmos, Submanifolds and holonomy, Chapman Hall/CRC Res. Notes in Math. 434, Chapman \& Hall/CRC, Boca Raton, 2003

[3] A. Borel, Essays in the history of Lie groups and algebraic groups, History of Mathematics, Vol. 21, American Mathematical Society, Providence 2001

[4] R. Bотт, The stable homotopy of the classical groups, Ann. Math. 70 (1959), 313-337

[5] B.-Y. Chen \& T. NAgano, Totally geodesic submanifolds of symmetric spaces II, Duke Math. J. 45 (1978), 405-425

[6] B.-Y. Chen \& T. NAGAno, A Riemannian geometric invariant and its applications to a problem of Borel and Serre, Trans. Am. Math. Soc. 308 (1988), 273-297

[7] J.-H. Eschenburg \& E. Heintze, Extrinsic symmetric spaces and orbits of $s$-representations, Manuscr. Math. 88 (1995), 517-524 
[8] J.-H. Eschenburg, P. Quast \& M. S. Tanaka, Isometries of hermitian symmetric spaces, J. Lie Theory 23 (2013), 113-118

[9] J.-H. Eschenburg, P. Quast \& M. S. Tanaka, Maximal tori of extrinsic symmetric spaces and meridians, Osaka J. Math., accepted for publication

[10] J.-H. Eschenburg, P. Quast \& M. S. Tanaka, Isometries of extrinsic symmetric spaces, in preparation

[11] D. Ferus, Immersionen mit paralleler zweiter Fundamentalform: Beispiele und Nicht- Beispiele., Manuscr. Math. 12 (1974), 153-162

[12] D. Ferus, Immersions with parallel second fundamental form, Math. Z. 140 (1974), 87-93

[13] D. Ferus, Produkt-Zerlegung von Immersionen mit paralleler zweiter Fundamentalform, Math. Ann. 211 (1974), 1-5

[14] D. Ferus, Symmetric submanifolds of euclidean space, Math. Ann. 247 (1980), 81-93

[15] S. Gindikin \& S. KAneYUKI, On the automorphism group of the generalized conformal structure of a symmetric $R$-space, Differ. Geom. Appl. 8 (1998), 21-33

[16] S. Helgason, Differential geometry, Lie groups and symmetric spaces, Pure Appl. Math. 80, Academic Press, New York, 1978

[17] S. Kobayashi, Isometric imbeddings of compact symmetric spaces, Tohoku Math. J. 20 (1968), 21-25

[18] S. Kobayashi \& T. Nagano, On filtered Lie algebras and geometric structures I, J. Math. Mech. 13 (1964), 875-907

[19] S. Kobayashi \& T. Nagano, On filtered Lie algebras und geometric structures II, J. Math. Mech. 14 (1965), 513-521

[20] D.S.P. LEunG, The reflection principle for minimal submanifolds of Riemannian symmetric spaces, J. Differ. Geom. 8 (1973), 153-160

[21] D.S.P. LEung, On the classification of reflective submanifolds of Riemannian symmetric spaces, Indiana Univ. Math. J. 24 (1974), 327-339

[22] D.S.P. Leung, Reflective submanifolds. III. Congruency of isometric reflective submanifolds and corrigenda to the classification of reflective submanifolds, J. Differ. Geom. 14 (1979), 167-177

[23] D.S.P. Leung, Reflective submanifolds. IV. Classification of real forms of hermitian symmetric spaces, J. Differ. Geom. 14 (1979), 179-185

[24] O. Loos, Symmetric spaces I: general theory, W. A. Benjamin, New York 1969

[25] O. Loos, Symmetric spaces II: compact spaces and classification, W. A. Benjamin, New York 1969

[26] O. Loos, Jordan triple systems, $R$-spaces and bounded symmetric domains, Bull. Amer. Math. Soc. 77 (1971), 558-561

[27] O. Loos, Charakterisierung symmetrischer Räume durch ihre Einheitsgitter, Math. Z. 189 (1985), $211-226$

[28] A.-L. Mare \& P. Quast, On some spaces of minimal geodesics in Riemannian symmetric spaces, Q. J. Math. 63 (2012), 681-694

[29] A.-L. Mare \& P. Quast, Bott periodicity for inclusions of symmetric spaces, Doc. Math., J. DMV 17 (2012), 911-952

[30] J. Milnor, Morse Theory, Annals of Mathematics Studies, Vol. 51, Princeton University Press, Princeton, 1969

[31] T. Nagano, Transformation groups on compact symmetric spaces, Trans. Amer. Math. Soc. 118 (1965), 428-453

[32] T. Nagano, The involutions of compact symmetric spaces, Tokyo J. Math. 11 (1988), 57-79

[33] F. Platzer \& P. Quast, Convexity of reflective submanifolds in special unitary groups, Tokyo J. Math., accepted for publication

[34] P. Quast, A pinching theorem for extrinsically symmetric submanifolds of euclidean space, Manuscr. Math. 115 (2004), 427-436

[35] P. Quast \& M. S. Tanaka, Convexity of reflective submanifolds in symmetric $R$-spaces, Tohoku Math. J. 64 (2012), 607-616 
[36] P. Quast, A geometric proof of a result of Takeuchi, Tohoku Math. J. 66 (2014), 427-434

[37] T. SAKaI, On cut loci of compact symmetric spaces, Hokkaido Math. J. 6 (1977), 136-161

[38] W. Strübing, Symmetric submanifolds of Riemannian manifolds, Math. Ann. 245 (1979), 37-44

[39] M. TAKeuchi, Cell decompositions and Morse equalities on certain symmetric spaces, J. Fac. Sci., Univ. Tokyo, Sect. I 12 (1965), 81-191

[40] M. Takeuchi \& S. Kobayashi, Minimal imbeddings of $R$-spaces, J. Differ. Geom. 2 (1968), 203-215

[41] M. TAKEUCHI, Stability of certain minimal submanifolds of compact hermitian symmetric spaces, Tohoku Math. J. 36 (1984), 293-314

[42] M. TAkeuchi, Basic transformations of symmetric R-spaces, Osaka J. Math. 25 (1988), 259-297

[43] M. S. TAnaka, Subspaces in the category of symmetric spaces, in M. Guest (ed.) et al.: Differential geometry and integrable systems, Tokyo 2000, Contemp. Math. 308 (2002), 305-313

[44] M. S. TANKA \& H. TASAKI, The intersection of two real forms in Hermitian symmetric spaces of compact type, J. Math. Soc. Japan 64 (2012), 1297-1332

[45] M. S. TANKA \& H. TASAKI, Antipodal sets of symmetric $R$-spaces, Osaka J. Math. 50 (2013), 161-169

[46] H. Tasaki, The intersection of two real forms in the complex hyperquadric, Tohoku Math. J. 62 (2010), 375-382

(Quast) Institut für Mathematik, Universität Augsburg, D 86135 Augsburg, Germany

E-mail address: peter.quast@math.uni-augsburg.de

(Tanaka) Faculty of Science and Technology, Tokyo University of Science, Noda, Chiba 278-8510, JAPAN

E-mail address: tanaka_makiko@ma.noda.tus.ac.jp 